\title{
Dynamic compressive strength of silicon carbide under uniaxial compression
}

\author{
Sai Sarva ${ }^{1}$, Sia Nemat-Nasser * \\ Center of Excellence for Advanced Materials, Department of Mechanical and Aerospace Engineering, University of California at San Diego, \\ 9500 Gilman Drive, La Jolla, CA 92093-0416 USA
}

\begin{abstract}
$\mathrm{SiC}$ is used in impact related applications, such as integrated armor. This necessitates development of models which describe its dynamic behavior and failure modes. Experimental results, which relate the strain rate to the compressive strength of SiC under uniaxial loading are obtained. Quasi-static tests are performed using a hydraulically driven Instron universal testing machine. Dynamic tests are performed using a modified split Hopkinson pressure bar. A marked increase is observed in the compressive strength at strain rates greater than $10^{2} \mathrm{~s}^{-1}$. The failure modes are similar in specimens loaded at both low and high strain rates. At the peak stress, the specimens fail by axial splitting. This paper compares the experimental results to those predicted by a simple model of interacting, dynamically growing, wing cracks which coalesce, leading to failure. (C) 2001 Elsevier Science B.V. All rights reserved.
\end{abstract}

Keywords: Silicon carbide; Dynamic compression; Axial splitting; Wing cracks

\section{Introduction}

The study of the dynamic behavior of ceramics has been of interest for the past decade. Efforts have been made to understand the dynamic behavior, damage evolution, and failure modes in ceramics. However, there still exists ambiguity over many aspects due to a lack of sufficient experimental data. Researchers have experimentally studied the dynamic response of ceramics under uniaxial and multiaxial compression. There have also been attempts to explain the failure phenomenon, by means of simple micromechanical models. The compressive failure strength of ceramics was first shown to be strain-rate sensitive by Lankford [1-3]. This phenomenon has since been studied in various ceramics. It has been suggested that there exists a critical strain rate (around $1000 \mathrm{~s}^{-1}$ ), above which the compressive strength is strongly strain-rate sensitive.

Horii and Nemat-Nasser [4-6] attribute the overall failure of ceramics to the coalescence of microcracks, originating from pre-existing flaws such as grain boundaries, pores, and inclusions. In compression, ten-

\footnotetext{
* Corresponding author.

E-mail address: sia@shiba.ucsd.edu (S. Nemat-Nasser).

${ }^{1}$ Tel.: + 1-858-534-4914 fax: + 1-858-534-2727
}

sile microcracks, which nucleate at defects, may grow unstably in the direction of the maximum compressive load. Nemat-Nasser and Horii [4] have given an exact solution, based on linear elastic fracture mechanics, to describe these tension cracks, also called wing cracks. Nemat-Nasser and Deng [7,8] have developed a simple model of an array of interacting dynamically growing wing cracks to describe the influence of the microstructure on the behavior of ceramics at high strain rates.

The object of the present research is to study the dynamic behavior of $\mathrm{SiC}$ under uniaxial compression, with the intent to further understand the relationship between the microstructure and the failure strength. The experimental results are compared to the NematNasser-Deng model.

\section{Experimental techniques}

\subsection{Material}

Hot-pressed sintered silicon carbide is used for the present research. The as-received material is machined into rectangular specimens of length $6.35 \mathrm{~mm}$ and cross-sectional area $3.5 \times 3.5 \mathrm{~mm}$. The opposite faces of the specimen are polished parallel, to an accuracy of 3 


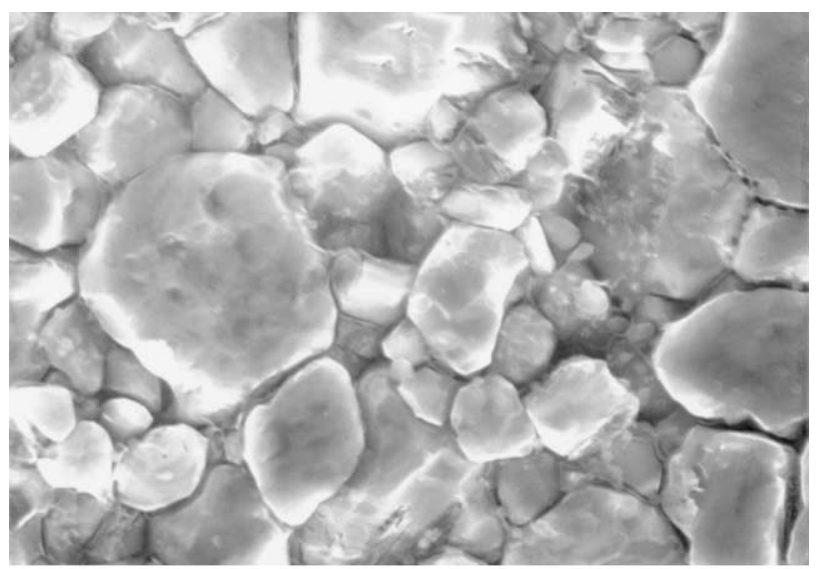

$10 \mu \mathrm{m}$

Fig. 1. Grain structure of as-received hot-pressed sintered SiC.

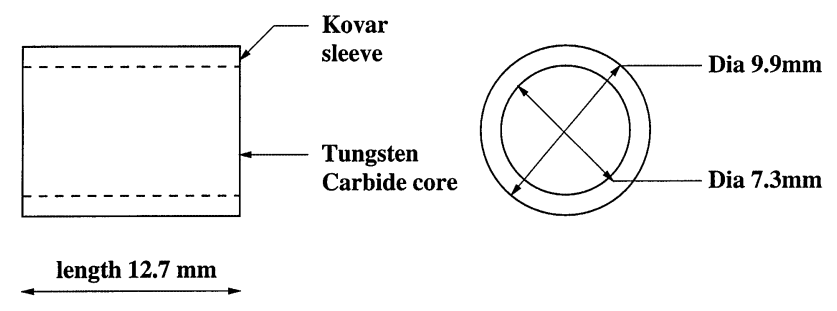

Fig. 2. Platen design.

$\mu \mathrm{m}$, in order to maintain uniaxial loading. Fig. 1 shows the microstructure of the as-received material. The grain size is approximately $6 \mu \mathrm{m}$. The porosity resulting from the sintering process can be seen at the grain boundaries.

\subsection{Quasi-static test procedures}

Quasi-static tests at strain rates ranging from $10^{-4}$ to $10^{-2} \mathrm{~s}^{-1}$ are performed on a servo-hydraulic Instron testing machine, of load capacity $89000 \mathrm{~N}$. A load cell is used to measure the axial load. To obtain accurate strain data, a strain gauge is attached to the specimen.

\subsection{Dynamic test procedures}

A modified split Hopkinson bar (MSHB) is used to perform high strain-rate tests. The MSHB incorporates new techniques developed at the Center of Excellence for Advanced Materials (CEAM) at UCSD. Like the conventional Hopkinson bar, it consists of a striker bar, an incident bar, and a transmission bar. The specimen is held between the incident and transmission bars. The striker bar is projected towards the incident bar, by means of a gas gun. On impact, an elastic compressive pulse is generated in the incident bar. When this pulse, also known as the incident pulse, reaches the sample, part of it is reflected and the remaining part is transmitted through the sample into the transmission bar. Strain gauges mounted on the bars measure the incident, reflected, and transmitted pulses. One-dimensional elastic wave equation relations are then used to calculate the strain in the sample from the reflected pulse and the corresponding stress from the transmitted pulse. For further details see Ref. [9]. The MSHB incorporates a momentum trap, which renders the Hopkinson bar capable of dynamic recovery experiments. The samples can thus be subjected to a single pulse and then recovered without being subjected to any additional loading.

Testing of ceramics is difficult because of their very high compressive strengths and low failure strains. In particular, it is important to ensure that a uniform state of stress is attained along the length of the sample. Hence, the following further modifications are made [10]:

1. A thin copper cushion is placed at the impact end of the incident bar. This metal cushion, also called the pulse-shaper, introduces a monotonically increasing ramp-like stress pulse in the incident bar [11-14]. Since the sample response is linear, essentially up to the failure stress, a ramp-like pulse is necessary to subject the sample to a constant strain rate during the test. In the present case, a ramp loading with a rise time of approximately $20 \mu \mathrm{s}$ is employed (see Section 3.1). The longitudinal wave speed for $\mathrm{SiC}$ is $10500 \mathrm{~m} \mathrm{~s}^{-1}$. Hence, for a sample of $6.35 \mathrm{~mm}$ length, the ramp loading ensures an essentially uniform state of stress along the sample length (to within less than $3 \%$ stress difference between two ends of the sample) throughout the test. The uniform state of stress is attained in less than $3 \mu \mathrm{s}$.

2. Platens made of tungsten carbide (WC) are used to prevent the indentation of the bars. However, WC itself is weaker than $\mathrm{SiC}$ and the platens often fail before the specimen does. Hence, these platens are further strengthened by confining them in a thin tube of Kovar. These platens are designed such that their impedance matches that of the bars. To verify that the platens are indeed impedance matched, dummy tests were performed. The platens were sandwiched between the bars and pulse propagation was observed. It was observed that the entire incident pulse was transmitted with negligible losses. Fig. 2 is the design of platens used.

3. The failure strains in ceramics are very low $(\approx 1 \%)$. The resulting reflected pulse is thus very weak and it is not an accurate measure of the strain of the sample. Therefore, strain gauges are glued onto the sample to precisely measure the sample strain [1114]

Using a MSHB of $12.7 \mathrm{~mm}$ diameter, the samples are dynamically tested over strain rates ranging from 250 to $1200 \mathrm{~s}^{-1}$. 


\section{Experimental results}

\subsection{Sample test}

Fig. 3 shows the time resolved voltage output received from strain gauges attached on the incident and transmission bars for a sample test. The failure stress is

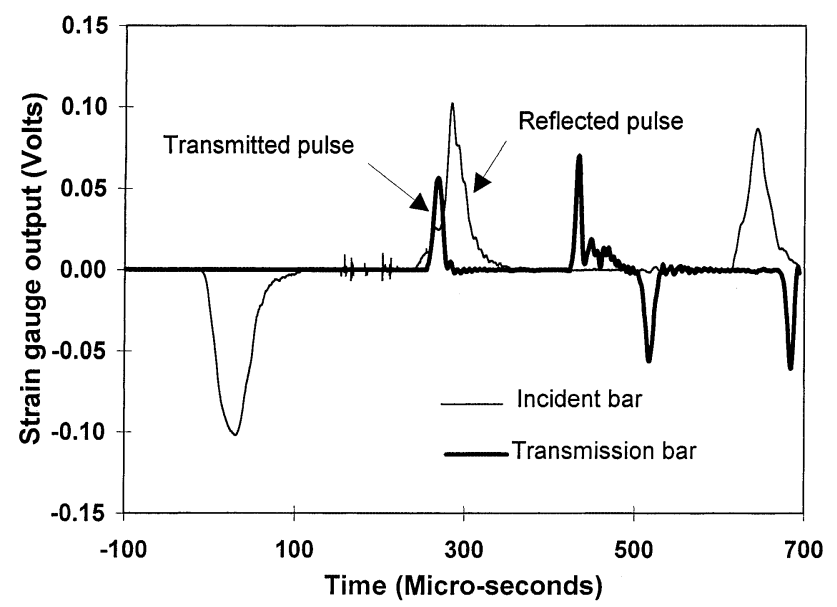

Fig. 3. Output obtained from strain gauges on the incident and transmission bars.

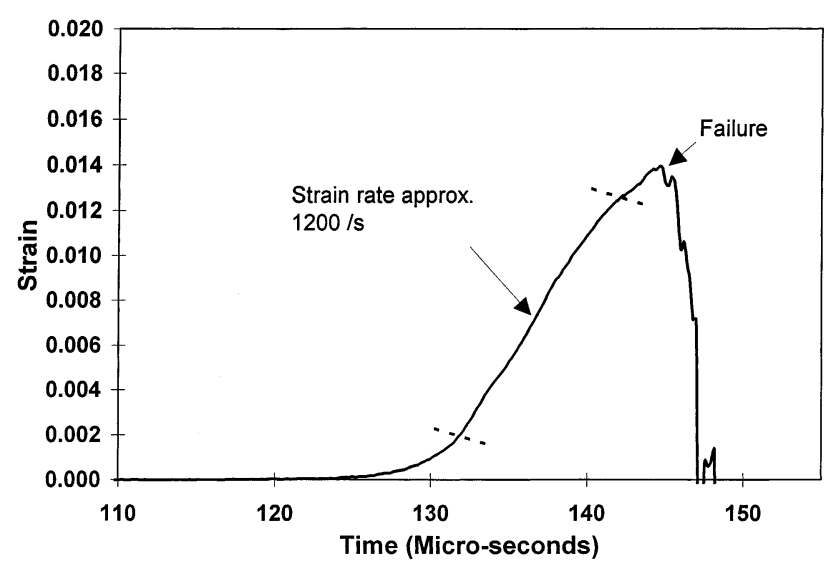

Fig. 4. Strain time profile obtained from strain gauge on the sample.

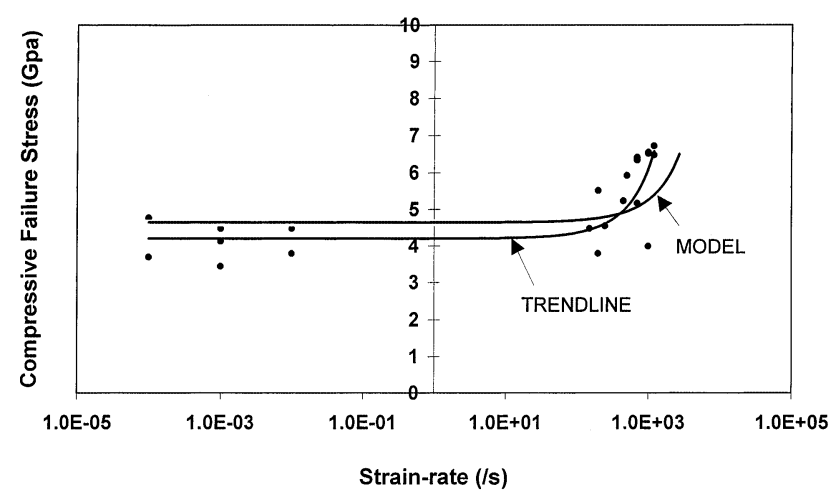

Fig. 5. Comparison of experimental results to the Nemat-NasserDeng model. calculated from the transmitted pulse. Fig. 4 is the strain-time profile calculated from the output received from the strain gauge attached onto the sample. The sample is loaded for approximately $20 \mu \mathrm{s}$, prior to failure. As can be seen from Fig. 4, the strain rate achieved for the sample test is about $1200 \mathrm{~s}^{-1}$.

\subsection{Compressive failure strength}

The experimental results obtained show the effect of the strain rate on the compressive failure strength of $\mathrm{SiC}$; see Fig. 5. As can be seen, the compressive strength of $\mathrm{SiC}$ under quasi-static testing conditions is approximately $4.2 \mathrm{GPa}$. Dynamic tests confirm the strain rate sensitivity of the compressive strength of this material. The strength increases from $4.2 \mathrm{GPa}$ at a strain rate of $250 \mathrm{~s}^{-1}$ to $7.0 \mathrm{GPa}$ at $1200 \mathrm{~s}^{-1}$. The stress-strain curves indicate a nearly linear response up to failure, for both static and dynamic tests, indicating very little plasticity.

\subsection{Failure modes}

Under both static and dynamic conditions, the samples failed by axial splitting. At the peak stress, the samples shattered to fragments. Fig. 6 compares the fragment size for static and dynamic tests. The fragment size for the static test can be seen to be visibly larger than that for a dynamic test. The fragment size is related to the crack size at coalescence. The observations confirm predictions by Grady and Kipp [15,16], that fragment size reduces with increasing strain rate.

\section{Comparison to the wing-crack array model}

Nemat-Nasser and Deng [7] seek to explain the relationship between the microstructure and the dynamic behavior by means of a simple wing-crack array model. They consider an infinite array of interacting wing cracks subjected to a dynamic farfield biaxial compressive load. Under suitable loading conditions, the tensile cracks grow unstably in the direction of the maximum compressive load. Coalescence of these tensile cracks results in failure. The dynamic stress intensity factor at the crack tips in the crack array is calculated by superposing the solution for a crack array under pairs of concentrated forces applied at the crack centers and the solution for crack array under uniform farfield stress. The functions, which represent the inertia effect during dynamic crack growth in the stress intensity factor, are then used to obtain a solution for the collinear wingcrack array. This gives an expression for the crack growth rate in terms of stress intensity factors for crack array under concentrated and uniform loads, which are further dependent on the microstructure. Since failure 

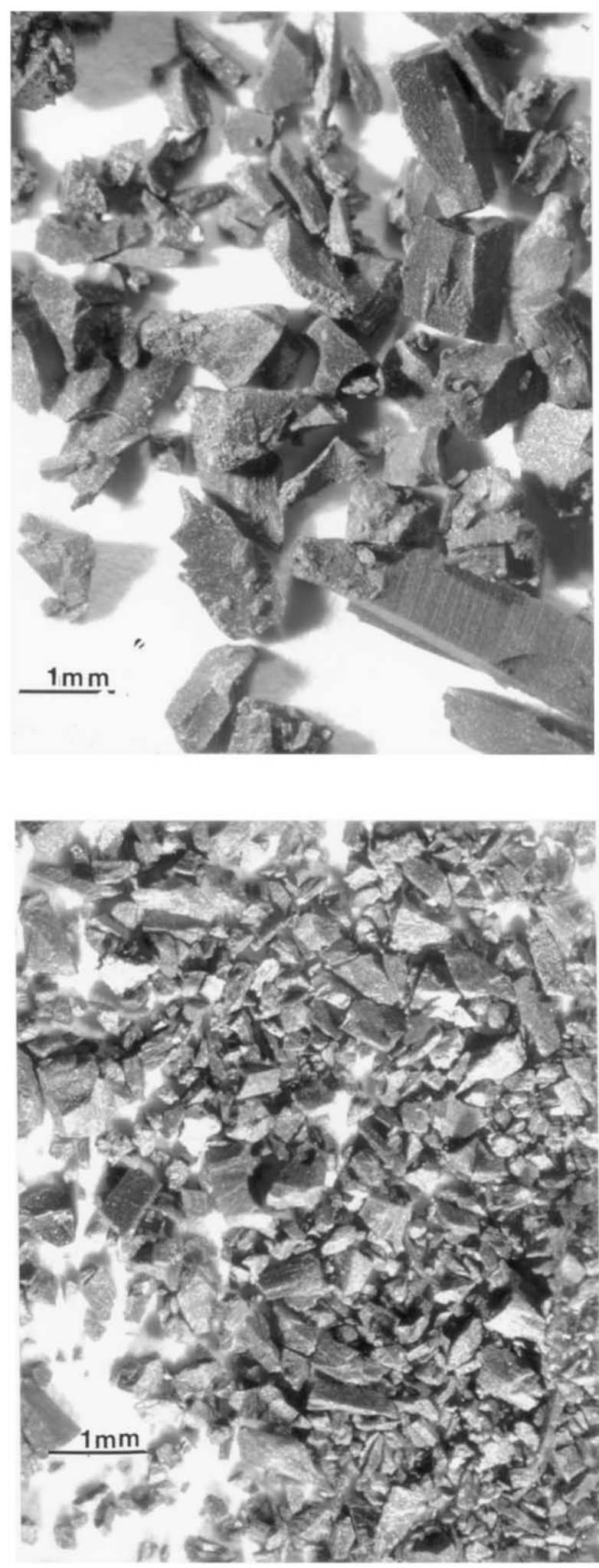

Fig. 6. Comparison of fragment size for static and dynamic tests.

occurs when the cracks grow and coalesce, the time to failure can be calculated from the crack growth rate and the crack length prior to failure. The crack length prior to failure is also the microflaw spacing. The failure stress is then calculated from the Young's modulus, the strain rate and the time to failure.

For the parametric study of the model, the material constants of $\mathrm{SiC}$ are taken as: Young's modulus $E=$ $470 \mathrm{GPa}$; Poisson's ratio $v=0.20$; density $\rho=3.2 \mathrm{~g}$ $\mathrm{cm}^{-3}$; fracture toughness $\bar{K}_{\mathrm{Ic}}=4.5 \mathrm{MPa} \mathrm{m}^{-2}$; effective Rayleigh wave speed $C_{\mathrm{R}}=1500 \mathrm{~m} \mathrm{~s}^{-1}$. It is expected that the damage caused due to wing-cracks reduces the Rayleigh wave speed of the material. Hence a wave speed, that is approximately third of an intact material is chosen. For the wing-crack, the co-efficient of friction $\mu=0.4$; Other parameters defining the wing crack are left as in [7].

The model includes the effect of the microstructure in terms of microflaw size and microflaw spacing. In ceramics, the exact mechanisms of flaw nucleation and the distance the cracks traverse prior to failure are not yet fully understood. For comparison purposes, the model is plotted for a microflaw size of $100 \mu \mathrm{m}$ and flaw spacing of $1.6 \mathrm{~mm}$. The experimental results have been compared to the model, under uniaxial loading conditions, in Fig. 5.

\section{Conclusions}

As expected, the compressive failure stress of $\mathrm{SiC}$ has been seen to be strain-rate sensitive. The critical strain rate above which the compressive strength becomes strongly rate sensitive is around $250 \mathrm{~s}^{-1}$. Under uniaxial compression, samples fail by axial splitting under both static and dynamic loading conditions. The size of the fragments occurring due to axial splitting is also strain-rate sensitive. The fragment size is considerably larger for static tests as compared to dynamic tests. Since fragment size is directly proportional to the length of the tensile cracks, it can be concluded that in a dynamic test the length of the microcracks is much smaller. This observation indicates that a many more microcracks are nucleated during a dynamic test. The mechanisms for strain rate sensitivity may be closely related to this phenomenon indicating that a detailed study of the fragment size is essential.

The Nemat-Nasser-Deng model [7] gives a qualitative description of the high-strain rate behavior of ceramics. The values of the microstructural parameters (flaw spacing $=1.6 \mathrm{~mm}$, flaw size $=100 \mu \mathrm{m}$ ), assumed to plot the model results, are nominal. However, the model does not account for the fact that the microcrack size for dynamic tests is smaller than that for static tests, as is manifested by the smaller fragment size. The model indicates that the crack growth velocity and dynamic stress intensity factor at the crack tips largely influence the overall dynamic behavior of ceramics. Further research is necessary so as to further understand the failure mechanisms and obtain experimental data in order to improve on the model. 


\section{Acknowledgements}

The authors thank Dr M. Beizai for his help with Fortran programming and Dr J. Shih for his help with material procurement. This project was supported by the US Army Research Office under Contract No DAAH04-96-1-0376, to the University of California at San Diego.

\section{References}

[1] J. Lankford, J. Am. Ceram. Soc. 64 (1981) C33-C44.

[2] J. Lankford, Int. J. Rock Mech. Miner. Sci. Geomech. Abstr. 18 (1981) 173.

[3] J. Lankford, J. Mater. Sci. 16 (1981) 1567.

[4] S. Nemat-Nasser, H. Horii, J. Geophys. Res. 87 (1982) 6805.

[5] H. Horii, S. Nemat-Nasser, J. Geophys. Res. 90 (1985) 3105.
[6] H. Horii, S. Nemat-Nasser, Phil. Trans. R. Soc. Lond. 319 (1986) 337.

[7] S. Nemat-Nasser, H. Deng, Acta Metall. Mater. 42 (3) (1994) 1013.

[8] H. Deng, S. Nemat-Nasser, Trans. ASME 116 (1994) 286.

[9] P. Follansbee, Metals Handbook, vol. 8, American Society for Metals, Materials Park, OH, 1985, p. 198.

[10] S. Nemat-Nasser, J.B. Isaacs, J.E. Starrett, Proc. R. Soc. Lond. A435 (1991) 371.

[11] W.P Rogers, J.B. Isaacs, S. Nemat-Nasser, in: D.B Thomson, D.E Chimenti (Eds.), Proceedings Review of Progress in Quantitative NDE, vol. 8B, Plenum, New York, 1989, p. 1827.

[12] W.P Rogers, S. Nemat-Nasser, J. Am. Ceram. Soc. 75 (1990) 136.

[13] V. Sharma, S. Nemat-Nasser, K.S. Vecchio, Exp. Mech. 34 (1994) 315.

[14] G. Subhash, S. Nemat-Nasser, J. Am. Ceram. Soc. 76 (1993) 153.

[15] D.E Grady, J. Appl. Phys. 53 (1982) 322.

[16] D.E Grady, M.E. Kipp, Int. J. Rock Mech. Miner. Sci. 17 (1980) 147. 